\title{
Selective enhancement of cellular oxidative stress by chloroquine: implications for the treatment of glioblastoma multiforme
}

\author{
Steven M. Toler, Pharm.D., Ph.D., Dennis Noe, M.D., and Amarnath Sharma, Ph.D. \\ Department of Clinical Pharmacology, Pfizer, Incorporated, 50 Pequot Avenue, \\ New London, Connecticut
}

\begin{abstract}
$\checkmark$ Chloroquine is used in the treatment of malaria, a disease caused by infection with the parasite Plasmodium. Although chloroquine appears to possess diverse pharmacological activity, its plasmodicidal activity results from augmentation of parasite oxidative stress. Chloroquine also appears to augment oxidative stress in metabolically active mammalian cells, including human astroglial cells. The authors propose that chloroquine may augment oxidative stress induced by radiotherapy in the treatment of glioblastoma multiforme, enhancing therapeutic efficacy. Such an effect would be consistent with the known pharmacological effects of chloroquine observed in Plasmodium. Other selective redox agents, such as tempol and artemisinin, should be investigated clinically for therapeutic benefit when coadministered with combined radio- and chemotherapy for cancer.
\end{abstract}

KEY WORDS • glioblastoma multiforme • oxidative stress • chloroquine

$\mathrm{G}$ LIOBLASTOMA multiforme is the most common and aggressive primary glial tumor, comprising $30 \%$ of all brain malignancies in adults. ${ }^{37}$ This is a rapidly infiltrating tumor that is resistant to current therapies, resulting in a median survival duration of approximately 1 year. ${ }^{37}$ Despite all efforts, few therapeutic advances have been achieved over the last decade that have significantly improved survival rates. Nevertheless, results from two small studies support the suggestion that the addition of chloroquine to conventional therapy may improve the median survival duration in patients with GBM. ${ }^{2,38}$

\section{Advances in Treatment of GBM}

In an initial study, a statistically significant increase in survival time was found following conventional therapy plus chloroquine ( $33 \pm 5$ months) relative to conventional therapy plus placebo $(11 \pm 2$ months, nine patients in each group). ${ }^{2}$ In a second follow-up study, the median survival time increased from 11 months following placebo plus standard therapy to 24 months following chloroquine plus standard therapy (15 patients in each group), and although these results failed to achieve statistical significance, there was a trend toward an increase in overall survival for the chloroquine treatment arm. ${ }^{38}$ At least two the-

Abbreviation used in this paper: $\mathrm{GBM}=$ glioblastoma multiforme. ories have been proposed to account for the apparent therapeutic benefit associated with chloroquine treatment: 1) chloroquine possesses antimutagenic properties that impede the development of treatment-resistant clones; and 2) the lysosomotrophic effects of chloroquine lead to cell necrosis. ${ }^{2,46}$ However, an alternative hypothesis, enhancement of cellular oxidative stress, may better explain the apparent improvement in median survival duration noted in patients undergoing concurrent chloroquine therapy.

\section{Importance of Oxidative Stress}

The treatment of patients with GBM typically includes surgical tumor ablation and radiotherapy with or without chemotherapy. Postoperative radiotherapy has been recognized as the standard of care for several decades, whereas the additional use of chemotherapy has been controversial. ${ }^{25}$ Recently, tumor ablation followed by radiotherapy and concurrent temozolomide administration has become the new standard of care. ${ }^{3}$ In the studies mentioned earlier (Briceno et al. and Sotelo et al.), chloroquine therapy was initiated 5 days postsurgery, prior to the start of radiotherapy (total dose $6000 \mathrm{~Gy}$ ) or chemotherapy (four cycles of carmustine, $200 \mathrm{mg} / \mathrm{m}^{2}$ ) in patients with GBM. $^{2,38}$

In tissues exposed to treatment, radiotherapy produces cytotoxic free radicals and extensive oxidative stress. ${ }^{6,8}$ Ionizing radiation produces a number of reactive oxygen species, including superoxide ion, hydroxyl radical, and hydrogen peroxide; it is currently postulated that the pri- 
mary therapeutic effects from radiotherapy are mediated through the generation of cytotoxic reactive oxygen species. ${ }^{6,22}$ Furthermore, cancer cells accumulate iron, and radiotherapy elevates intracellular free iron concentrations that can lead to the production of cytotoxic hydroxyl radicals through the Fenton reaction. ${ }^{19,41}$ Therefore, cells exposed to radiotherapy are subjected to heightened oxidative stress. These cells must either adapt to such conditions or perish. Adaptation often includes induction of biochemical pathways that quench or mediate the destructive effects of reactive intermediates. In U251 human glioblastoma cells, radiation has been shown to induce the expression of a number of antioxidant enzymes, including superoxide dismutase (twofold) and glutathione peroxidase (fivefold). ${ }^{22}$ Antioxidant adaptations noted in U251 human glioblastoma cells are consistent with clinical observations of GBM resistance to radiotherapy.,22

\section{Chloroquine Enhances Oxidative Stress}

Chloroquine, a 9-aminoquinoline synthesized in 1934, is used in the treatment of malaria, a disease caused by infection with the parasite Plasmodium. Although chloroquine appears to possess diverse pharmacological activities, its plasmodicidal effect results from augmentation of oxidative stress in this parasite. ${ }^{10,13,26}$ During schizogony, Plasmodium subsists on amino acids derived from hemoglobin, resulting in production of the prooxidant heme iron complex ferriprotoporphyrin IX. This heme iron complex is detoxified in Plasmodium through a number of glutathione-dependent pathways (including incorporation into hemozoin). ${ }^{13}$ Chloroquine appears to inhibit the detoxification of ferriprotoporphyrin IX, in part by diminishing the availability of reduced glutathione., ${ }^{71}$ Excess iron and ferriprotoporphyrin IX, in the absence of sufficient glutathione, leads to autooxidation and parasite death. Furthermore, chloroquine appears to interact directly with free ferriprotoporphyrin IX in an electron transfer reaction that produces highly reactive radicals. ${ }^{26}$ Chloroquine also appears to augment oxidative stress in mammalian cells that have high metabolic activity, and it generates reactive oxygen species in human astroglial cells. ${ }^{1,21,27,31,32,40,42}$

\section{Therapeutic Benefit of Chloroquine: Potential Mechanisms}

We propose that chloroquine may augment oxidative stress induced by radiotherapy in cancer cells. Such an effect would be consistent with the known pharmacological effects of chloroquine in Plasmodium. ${ }^{26}$ Directed radiotherapy selectively produces oxidative stress in targeted cancer cells, generating free radicals and elevating concentrations of free iron. ${ }^{41}$ Following such treatment, cancer cells often induce antioxidant defenses as an adaptive response to thwart cytotoxicity. Agents such as chloroquine that promote oxidative stress may hinder the effectiveness of these antioxidant adaptations, resulting in heightened tumoricidal activity. The therapeutic benefit noted with chloroquine appears to be the result of effective proliferation of reactive intermediates initiated by radiotherapy, resulting in increased tumor cytotoxicity.

At least two alternative theories have been proposed to explain the apparent benefit of adding chloroquine to traditional GBM therapy. One theory contends that chloro- quine is antimutagenic and prevents the development of treatment-resistant clones. ${ }^{2}$ However, there are at least two inconsistencies with this theory. First, there is little evidence that chloroquine is antimutagenic. Although quinacrine, a compound structurally related to chloroquine, has demonstrated antimutagenic properties in two cell lines, it appears to be mutagenic in others. ${ }^{12,15,44}$ Furthermore, most data on chloroquine support its mutagenic properties rather than antimutagenic effects, which is consistent with other 4-aminoquinolines. ${ }^{4,9,29,44}$ Second, if chloroquine possessed antimutagenic properties, diminished rather than enhanced efficacy might be expected with concurrent radio- and chemotherapy, because treatment-induced mutations are what yield the desired tumor cytotoxicity.

Another theory states that the radiosensitization produced by chloroquine is due to its lysosomotrophic effects. ${ }^{46}$ The lysosomotrophic effects produced by chloroquine, a cationic amphipathic drug, result from protonation and subsequent molecular "trapping" within acidic lysosomes, which elevates lysosomal $\mathrm{pH}$. However, there are vast numbers of cationic amphipathic drugs that have not been reported to induce radiosensitization (Table 1). The radiosensitizing effects of chloroquine appear to be unique relative to other lysosomotrophic compounds like ammonium chloride, fluoxitine, and amitriptyline. Although lysosomal membrane rupture contributes to cell necrosis after radiation and chloroquine treatment, the cause of membrane rupture may be lysosomal membrane lipid peroxidation produced by the distinct prooxidant properties of chloroquine rather than lysosomotrophism..$^{24,30,39}$

\section{Discussion}

Cancer cells, including malignant glial cells, accumulate iron to a greater extent than do normal cells, producing a condition similar to that found in Plasmodium. ${ }^{19,28}$ After radiotherapy, protein-bound intracellular iron is released as free iron, and chloroquine may promote oxidative stress through propagation of reactive oxygen species in a manner similar to the stress produced in Plasmodium. Of interest, the antimalarial agent artemisinin has recently demonstrated activity against human glioma and breast cancer cell lines in vitro. ${ }^{18}$ Artemisinin, an endoperoxide, appears to be activated to release reactive oxygen species by elevated concentrations of intracellular free iron in both Plasmodium and cancer cell lines. ${ }^{17,20}$ Another redoxactive compound, tempol (a piperidine nitroxide), demonstrates antiproliferative effects in C6 glioma cells in vitro and in mouse xenografts. ${ }^{11}$ Tempol, a superoxide dismutase mimetic, converts superoxide into hydrogen peroxide, which in the presence of free iron is converted to the highly reactive hydroxide ion. ${ }^{5}$ Like chloroquine, tempol has demonstrated plasmodicidal activity in vitro, further supporting theories about its role as a prooxidant agent in the presence of available iron. ${ }^{36}$ Tempol, artimisinin, and chloroquine appear to demonstrate little cytotoxicity in the absence of preexisting oxidative stress, thus sparing normal cells. ${ }^{11,34}$

Because it appears that chloroquine serves as a promoter rather than an initiator of oxidative stress, it may also prove beneficial in cases in which oxidative stress is ther- 


\section{TABLE 1}

Selected cationic amphipathic drugs/lysosomotrophic agents not associated with radio- or chemosensitization

\begin{tabular}{ll}
\hline \multicolumn{1}{c}{ Agent } & \multicolumn{1}{c}{ Pharmacological Class } \\
\hline $\begin{array}{l}\text { diphenhydramine } \\
\text { chlorphentermine }\end{array}$ & $\mathrm{H}_{1}$ blocker \\
fluoxitine & selective serotonin reuptake inhibitor \\
ammonium chloride & alkalinizing agent \\
amitriptyline & tricyclic antidepressant \\
propranolol & beta-blocker \\
chlorpromazine & dopamine antagonist \\
perhexaline & carnitine palmitoyltransferase inhibitor \\
amantadine & indirect dopamine agonist \\
gentamicin & aminoglycoside antibiotic \\
\hline
\end{tabular}

apeutically induced by cytotoxic agents. Tumor-selective chemotherapeutic regimens (such as emodin/arsenic trioxide) that preferentially induce heightened oxidative stress in cancer cells might exhibit additional efficacy if coadministered with chloroquine. ${ }^{45}$ Similarly, chloroquine may prove beneficial in the treatment of tumors characterized by phenotypic autooxidations, such as melanoma. ${ }^{16,33,35}$ Both pre- and posttreatment, malignant melanoma cells exhibit heightened oxidative stress. ${ }^{23,35,43}$ Preclinically, chloroquine increases cytotoxicity in melanoma cell lines when administered alone or following radiation or hyperthermia. ${ }^{14}$ Therefore, chloroquine therapy might prove beneficial in the treatment of malignant melanoma.

Because of its ability to induce oxidative stress in Plasmodium, chloroquine has been used in the treatment of malaria for nearly half a century. Other chemically and pharmacologically diverse agents, such as artemisinin and tempol, demonstrate plasmodicidal and tumoricidal activities, providing evidence for a link between therapeutic effect and the creation of oxidative stress. These prooxidant activities probably explain the beneficial effects noted with chloroquine in the treatment of GBM with traditional radiotherapy. By selectively promoting tumor oxidative stress, chloroquine could prove beneficial in the treatment of other tumor types such as malignant melanoma, or following chemotherapy. Other selective redox agents, such as tempol and artemisinin, should be investigated clinically for their possible therapeutic benefit when coadministered with traditional GBM regimens.

\section{References}

1. Balo KP, Mensah A, Mihluedo H: [Chloroquine maculopathy and prevention of malaria.] J Fr Ophtalmol 19:770-776, 1996 [Fr]

2. Briceno E, Reyes S, Sotelo J: Therapy of glioblastoma multiforme improved by the antimutagenic chloroquine. Neurosurg Focus 14(2): E3, 2003

3. Chamberlain MC: Treatment options for glioblastoma. Neurosurg Focus 20(4): E2, 2006

4. Chatterjee T, Muhkopadhyay A, Khan KA, Giri AK: Comparative mutagenic and genotoxic effects of three antimalarial drugs, chloroquine, primaquine and amodiaquine. Mutagenesis 13:619-624, 1998

5. Chen YF, Cowley AW Jr, Zou AP: Increased H(2)O(2) counteracts the vasodilator and natriuretic effects of superoxide dismutation by tempol in renal medulla. Am J Physiol Regul Integr Comp Physiol 285:827-833, 2003
6. Cook JA, Gius D, Wink DA, Krishna MC, Russo A, Mitchell JB: Oxidative stress, redox, and the tumor microenvironment. Semin Radiat Oncol 14:259-266, 2004

7. Deslauriers R, Butler K, Smith IC: Oxidant stress in malaria as probed by stable nitroxide radicals in erythrocytes infected with Plasmodium berghei. The effects of primaquine and chloroquine. Biochim Biophys Acta 931:267-275, 1987

8. Doroshenko N, Doroshenko P: The glutathione reductase inhibitor carmustine induces an influx of $\mathrm{Ca} 2+$ in $\mathrm{PC} 12$ cells. Eur J Pharmacol 497:17-24, 2004

9. Espinosa-Aguirre JJ, Aroumir C, Meza MT, Cienfuegos E, Cortina de Nava C: Genotoxicity of amebicide and anthelmintic drugs in Escherichia coli pol A+/pol A-. Mutat Res 188: 111-120, 1987

10. Farombi EO, Shyntum YY, Emerole GO: Influence of chloroquine treatment and Plasmodium falciparum malaria infection on some enzymatic and non-enzymatic antioxidant defense indices in humans. Drug Chem Toxicol 26:59-71, 2003

11. Gariboldi MB, Ravizza R, Petterino C, Castagnaro M, Finocchiaro G, Monti E: Study of in vitro and in vivo effects of the piperidine nitroxide Tempol-a potential new therapeutic agent for gliomas. Eur J Cancer 39:829-837, 2003

12. Gasc AM, Sicard AM: Genetic studies of acridine-induced mutants in Streptococcus pneumoniae. Genetics 90:1-18, 1978

13. Ginsburg H, Golenser J: Glutathione is involved in the antimalarial action of chloroquine and its modulation affects drug sensitivity of human and murine species of Plasmodium. Redox Rep 8:276-279, 2003

14. Inoue S, Hasegawa K, Ito S, Wakamatsu K, Fujita K: Antimelanoma activity of chloroquine, an antimalarial agent with high affinity for melanin. Pigment Cell Res 6:354-358, 1993

15. Johnson HG, Bach MK: Apparent antimutagenic activity of quinacrine hydrochloride in Detroit-98 human sternal marrow cells grown in culture. Cancer Res 29:1367-1370, 1969

16. Joshi G, Sultana R, Tangpong J, Cole MP, St Clair DK, Vore $\mathrm{M}$ : Free radical mediated oxidative stress and toxic side effects in brain induced by the anti cancer drug adriamycin: insight into chemobrain. Free Radic Res 39:1147-1154, 2005

17. Kamchonwongpaisan S, Meshnick SR: The mode of action of the antimalarial artemisinin and its derivatives. Gen Pharmacol 27:587-592, 1996

18. Kim SJ, Kim MS, Lee JW, Lee CH, Yoo H, Shin SH: Dihydroartemisinin enhances radiosensitivity of human glioma cells in vitro. J Cancer Res Clin Oncol 132:129-135, 2006

19. Kwok JC, Richardson DR: The iron metabolism of neoplastic cells: alterations that facilitate proliferation? Crit Rev Oncol Hematol 42:65-78, 2002

20. Lai H, Sasaki T, Singh NP: Targeted treatment of cancer with artemisinin and artemisinin-tagged iron-carrying compounds. Expert Opin Ther Targets 9:995-1007, 2005

21. Lai WW, Lam DS: Chloroquine-induced bull's eye maculopathy. Hong Kong Med J 11:55-57, 2005

22. Lee HC, Kim DW, Jung KY, Park IC, Park MJ, Kim MS, et al: Increased expression of antioxidant enzymes in radioresistant variant from U251 human glioblastoma cell line. Int J Mol Med 13:883-887, 2004

23. Meyskens FL Jr, McNulty SE, Buckmeier JA, Tohidian NB, Spillane TJ, Kahlon RS: Aberrant redox regulation in human metastatic melanoma cells compared to normal melanocytes. Free Radic Biol Med 31:799-808, 2001

24. Michihara A, Toda K, Kubo T, Fujiwara Y, Akasaki K, Tsuji H: Disruptive effect of chloroquine on lysosomes in cultured rat hepatocytes. Biol Pharm Bull 28:947-951, 2005

25. Mirimanoff RO, Gorlia T, Mason W, Van den Bent MJ, Kortmann RD, Fisher B: Radiotherapy and temozolomide for newly diagnosed glioblastoma: recursive partitioning analysis of the EORTC 26981/22981-nCIC CE3 phase III randomized trial. J Clin Oncol 24:2563-2569, 2006

26. Monti D, Basilico N, Parapini S, Pasini E, Olliaro P, Taramelli 
D: Does chloroquine really act through oxidative stress? FEBS Lett 522:3-5, 2002

27. Murugavel P, Pari L: Attenuation of chloroquine-induced renal damage by alpha-lipoic acid: possible antioxidant mechanism. Ren Fail 26:517-524, 2004

28. Mykhaylyk O, Dudchenko N, Cherchenko A, Rosumenko V, Zozulya Y: Dysregulation of non-heme iron metabolism in glial brain tumors. Med Princ Pract 14:221-229, 2005

29. Obaseiki-Ebor EE, Obasi EE: Aspects of chloroquine mutagenicity. Mutat Res 175:51-59, 1986

30. Ollinger K, Brunk UT: Cellular injury induced by oxidative stress is mediated through lysosomal damage. Free Radic Biol Med 19:565-574, 1995

31. Pari L, Murugavel P: Protective effect of alpha-lipoic acid against chloroquine-induced hepatotoxicity in rats. J Appl Toxicol 24:21-26, 2004

32. Park J, Choi K, Jeong E, Kwon D, Benveniste EN, Choi C: Reactive oxygen species mediate chloroquine-induced expression of chemokines by human astroglial cells. Glia 47:9-20, 2004

33. Pavel S, van Nieuwpoort F, van der Meulen H, Out C, Pizinger $\mathrm{K}$, Cetkovska P, et al: Disturbed melanin synthesis and chronic oxidative stress in dysplastic naevi. Eur J Cancer 40: 1423-1430, 2004

34. Ravizza R, Cereda E, Monti E, Gariboldi MB: The piperidine nitroxide Tempol potentiates the cytotoxic effects of temozolomide in human glioblastoma cells. Int J Oncol 25:1817-1822, 2004

35. Sander CS, Hamm F, Elsner P, Thiele JJ: Oxidative stress in malignant melanoma and non-melanoma skin cancer. $\mathbf{B r} \mathbf{J}$ Dermatol 148:913-922, 2003

36. Schwartz E, Samuni A, Friedman I, Hempelmann E, Gokenser $\mathrm{J}$ : The role of superoxide dismutation in malaria parasites. Inflammation 23:361-370, 1999

37. Sheleg SV, Korotkevich EA, Zhavrid EA, Muravskaya GV, Smeyanovich AF, Shanko YG: Local chemotherapy with cisplatin-depot for glioblastoma multiforme. J Neurooncol 60: 53-59, 2002

38. Sotelo J, Briceno E, Lopez-Gonzalez MA: Adding chloroquine to conventional treatment for glioblastoma multiforme: a ran- domized, double-blind, placebo-controlled trial. Ann Intern Med 144:337-343, 2006

39. Sugioka Y, Suzuki M: The chemical basis for the ferriprotoporphyrin IX-chloroquine complex induced lipid peroxidation. Biochim Biophys Acta 1074:19-24, 1991

40. Toler SM: Oxidative stress plays an important role in the pathogenesis of drug-induced retinopathy. Exp Biol Med (Maywood) 229:607-615, 2004

41. Weijl NI, Elsendoorn TJ, Moison RM, Lentjes EG, Brand R, Berger R, et al: Non-protein bound iron release during chemotherapy in cancer patients. Clin Sci (Lond) 106: 475-484, 2004

42. Wielgo-Polanin R, Lagarce L, Gautron E, Diquet B, LaineCessac P: Hepatotoxicity associated with the use of a fixed combination of chloroquine and proguanil. Int J Antimicrob Agents 26:176-178, 2005

43. Woiniak A, Drewa G, Wozniak B, Schachtschabel DO, MilaKiersenkowska C, Drewa T: The effect of antitumor drugs on oxidative stress in B16 and S91 melanoma cells in vitro. Med Sci Monit 11: BR22-29, 2005

44. Xamena N, Creus A, Velazquez A, Marcos R: Testing of chloroquine and quinacrine for mutagenicity in Drosophila melanogaster. Mutat Res 158:177-180, 1985

45. Yang J, Li H, Chen YY, Wang XJ, Shi GY, Hu QS: Anthraquinones sensitize tumor cells to arsenic cytotoxicity in vitro and in vivo via reactive oxygen species-mediated dual regulation of apoptosis. Free Radic Biol Med 37:2027-2041, 2004

46. Zhao H, Cai Y, Santi S, Lafrenie K, Lee H: Chloroquine-mediated radiosensitization is due to the destabilization of the lysosomal membrane and subsequent induction of cell death by necrosis. RadiatRes 164:250-257, 2005

Manuscript received October 3, 2006.

Accepted in final form October 25, 2006.

Address reprint requests to: Steven M. Toler, Pharm.D., Ph.D., Department of Clinical Pharmacology, Pfizer Incorporated, $50 \mathrm{Pe}-$ quot Avenue, MS 6025-a3234, New London, Connecticut 06320. email: steven.m.toler@pfizer.com. 